\title{
Role of fibronectin in staphylococcal colonisation of fibrin thrombi and plastic surfaces
}

\author{
P. VALENTIN-WEIGAND, K. N. TIMMIS and G. S. CHHATWAL
}

Department of Microbiology, National Research Center for Biotechnology (GBF), Technical University, 3300 Braunschweig, Germany

\begin{abstract}
Summary. The adhesive glycoprotein fibronectin has been proposed as a mediator of adherence of certain gram-positive cocci to host cells and fibrin thrombi. This study compared the role of soluble and immobilised fibronectin in the adherence of coagulasenegative staphylococci (CNS) and Staphylococcus aureus to fibrin thrombi and plastic surfaces. Adherence of $S$. epidermidis to fibrin thrombi was significantly reduced when fibronectin was removed from the plasma used for thrombus preparation. Adherence was restored through restitution of fibronectin. S. epidermidis also adhered substantially more to plastic surface coated with fibronectin than to non-coated plastic. Increased adherence of CNS to plastic was also observed after coating with the $29-\mathrm{kDa}$ N-terminal fragment of fibronectin. Soluble fibronectin did not affect the adherence of CNS to fibrin thrombi or plastic surfaces. The adherence of $S$. aureus to fibrin thrombi was significantly increased by the addition of soluble fibronectin, but not by incorporation of fibronectin into the clot. These results indicate that the binding of fibronectin is an important factor in the adherence of staphylococci to fibrin clots and plastic surfaces and, thus, colonisation of these surfaces. However, the two species of staphylococci seem to employ different mechanisms of fibronectin-mediated adherence: $S$. epidermidis interacts mainly with fibronectin incorporated in fibrin clots or immobilised on implanted synthetic materials, whereas $S$. aureus adheres to the fibrin matrix through binding of soluble fibronectin present in wound exudates.
\end{abstract}

\section{Introduction}

Coagulase-negative staphylococci (CNS) have emerged as important nosocomial pathogens. Among CNS, Staphylococcus epidermidis is the species most frequently isolated from clinically relevant infections. ${ }^{1}$ Coagulase-positive $S$. aureus is a major wound pathogen in man, and accounts for $50 \%$ of the bacteraemias caused by the insertion of peripheral catheters. ${ }^{2,3}$

It is generally accepted that staphylococcal virulence is related to its ability to adhere to, and subsequently colonise, host tissues or implanted materials. Bacterial adherence represents, in most cases, a specific interaction between so-called adhesins of the bacterial cell wall and complementary structures on the eukaryotic target cell. ${ }^{4}$ The factors that mediate staphylococcal adherence and allow subsequent colonisation are still poorly defined. Surfaces to which staphylococci must adhere to initiate infections are primarily the fibrin clots formed immediately at an injured site or on the surfaces of inserted catheters and post-operative wounds, and the plasma-coated surfaces of implanted prosthetic devices.

Fibronectin is a major glycoprotein component

Received 10 April 1992; accepted 18 June 1992. incorporated into the fibrin matrix during formation of blood clots $^{5}$ and also constitutes a substantial component of the plasma proteins that rapidly coat synthetic materials after implantation. ${ }^{6}$ Certain staphylococcal and streptococcal species are capable of interacting with soluble plasma fibronectin as well as with fibronectin immobilised on host cells or synthetic materials ${ }^{7,8}$ and it has been suggested that these interactions might play a central role in the adherence of gram-positive cocci. ${ }^{9,10}$ However, studies on the adherence mechanisms of $S$. epidermidis, have focused mainly on the role of the extracellular slime substance and not on fibronectin, since CNS have little or no affinity for soluble fibronectin. ${ }^{11,12}$ On the other hand, it is known that fibronectin in a soluble form is different from fibronectin incorporated into a clot or immobilised on to a surface. ${ }^{13}$ Conflicting data have been published concerning the contribution of fibronectin to the adherence of $S$. aureus to fibrin clots. Toy et al. ${ }^{14}$ reported that $S$. aureus adhered to fibronectin incorporated into fibrin thrombi but did not find any effects of fibronectin on CNS adherence to fibrin clots. However, other studies indicated no substantial contribution of incorporated fibronectin to the adherence of $S$. aureus to fibrin thrombi. ${ }^{15,16}$ This prompted the present study, in which the role of 
soluble and immobilised fibronectin in the adherence of CNS and $S$. aureus to fibrin clots and plastic surfaces were compared.

\section{Material and methods}

\section{Bacteria}

A total of 34 staphylococcal strains was used in the study. Of these, 20 were clinical isolates from blood cultures or vaginal smears, comprising 10 each of $S$. aureus and S. epidermidis, which were identified by the API-Staph system (bioMérieux, Marcy-l'Etoile, France). The other 14 strains were CNS obtained from the German Culture Collection (DSM) and comprised two each of the species $S$. haemolyticus (DSM 20263, 20264), S. warneri (DSM 20036, 20316), S. hominis (DSM 20328, 20329), S. cohnii (DSM 20260, 20261), S. xylosus (DSM 20266, 20267), S. simulans (DSM 20322, 20323) and S. saprophyticus (DSM 20038, 20229). In addition, 12 clinical isolates, eight of Streptococcus pyogenes and two each of $S$. intermedius and Str. agalactiae, were included in this study. All bacteria were cultured in Todd-Hewitt Broth (Oxoid) for $18 \mathrm{~h}$ at $37^{\circ} \mathrm{C}$ and harvested by centrifugation $(20 \mathrm{~min}, 10000 \mathrm{~g})$. After washing twice in $0.15 \mathrm{M}$ phosphate-buffered saline (PBS), pH 7.5, bacterial suspensions containing $10^{9} \mathrm{cells} / \mathrm{ml}$ were prepared.

\section{Binding assays}

These were performed essentially as described previously for streptococci. ${ }^{17}$ Fibronectin was purified from human plasma by affinity chromatography according to the method of Miekka et al..$^{18}$ and labelled with ${ }^{125} \mathrm{I}$ as described by Hunter and Greenwood. ${ }^{19}$ The specific activity of labelled fibronectin was $3.15 \mathrm{mCi} / \mathrm{mg}$ of fibronectin. Binding was performed with $2 \times 10^{8}$ staphylococci and $15 \mathrm{ng}$ of labelled fibronectin for $45 \mathrm{~min}$ at room temperature. Binding buffer was PBS containing Tween $200.05 \%$.

\section{Adherence experiments}

Bacterial adherence to fibrin clots and plastic surfaces was quantified in a fluorometric micro-assay which was developed recently in our laboratory. ${ }^{20}$ The role of incorporated fibronectin in staphylococcal adherence to fibrin clots was studied by preparing clots from either untreated human plasma, plasma which had been depleted of fibronectin by affinity chromatography over a gelatin-agarose column, or depleted plasma which had been repleted with fibronectin. Bacteria were labelled with FITC ${ }^{20}$ and added to the clots at $10^{8}$ cells/well. Non-adherent bacteria were removed after 45 min by washing, and adherence was quantified by fluoroscanning as described previously. ${ }^{16}$ Preliminary studies showed that labelling of staphylococci with FITC had no significant effects on their fibronectin binding and adherence capabilities.
To determine staphylococcal adherence to plastic surfaces, FITC-labelled staphylococci $\left(10^{8}\right.$ in $100 \mu$ l of PBS/well) were added directly to microtitration plates (Nunc, Roskilde, Denmark) which had been coated with different amounts of purified fibronectin $\left(0.04-25 \mu \mathrm{g} /\right.$ well) or PBS for $16 \mathrm{~h}$ at $4{ }^{\circ} \mathrm{C}$, followed by treatment with PBS or PBS containing bovine serum albumin (PBS-BSA) $1 \%$ for $1 \mathrm{~h}$ to block non-specific attachment. After incubation for $1 \mathrm{~h}$ at $37^{\circ} \mathrm{C}$, the wells were washed four times with PBS and the plates were fluoroscanned. ${ }^{16}$ In parallel experiments, plates which had been coated with different amounts of BSA $\left(0.01-10000 \mu \mathrm{g} /\right.$ well, $16 \mathrm{~h}$ at $\left.4{ }^{\circ} \mathrm{C}\right)$ and then treated with indicated concentrations of fibronectin before determining adherence were used. To evaluate the role of soluble fibronectin in staphylococcal adherence, $10^{9}$ staphylococci were incubated with $100 \mu \mathrm{g}$ of purified human plasma fibronectin for $45 \mathrm{~min}$ at room temperature, washed with PBS, sonicated for $3 \mathrm{~s}$ to disrupt clumps and used for adherence tests. All experiments were done in quadruplicate and results were expressed as mean numbers (and SD) of adherent bacteria.

\section{Quantification of proteins bound to plastic surfaces by ELISA}

These studies were designed to determine the efficiency of fibronectin coating of non-coated and BSAcoated plastic surfaces, as well as to determine the degree of binding of fibronectin, fibrinogen, albumin and $\alpha_{2}$-macroglobulin to plastic surfaces after plasma coating. Microtitration plates were coated with albumin, fibronectin (as described earlier), human plasma (50 $\mu \mathrm{l} /$ well) or PBS (control) for $16 \mathrm{~h}$ at $4{ }^{\circ} \mathrm{C}$, washed twice with PBS, and non-coated surfaces were blocked with PBS-BSA or PBS-Tween $(0 \cdot 1 \%)$ for $1 \mathrm{~h}$ at $37^{\circ} \mathrm{C}$. After washing twice with PBS, bound proteins were detected with peroxidase-labelled antibodies against fibronectin, fibrinogen, albumin or $\alpha_{2}$ macroglobulin (diluted 1 in 500 in PBS; DAKO, Hamburg, Germany). After washing four times in PBS, reactions were visualised by adding substrate solution $(9 \mathrm{~mm}$ 3-dimethylaminobenzoic acid and 0.6 mM 3-methyl-2benzothiazolinon-hydrazone-hydrochloride-hydrate in $0.1 \mathrm{M}$ citric phosphate buffer, $\mathrm{pH} 5.0$, containing $\left.\mathrm{H}_{2} \mathrm{O}_{2} \quad 0.03 \%\right) 100 \mu \mathrm{l} /$ well. Reactions were stopped after $10 \mathrm{~min}$ with $4 \mathrm{~mm}$ sodium azide and plates were read at $540 \mathrm{~nm}$ in a microplate autoreader (Bio-Tek Instruments, Winooski, VT, USA). Results were expressed as mean (and SD) $A_{540}$ readings obtained from quadruplicate experiments. In parallel, standard curves were constructed by coating plates with twofold dilutions of the respective proteins.

\section{Adherence experiments with fibronectin fragments}

Purified fibronectin was fragmented by thrombin digestion into a $29-\mathrm{kDa} \mathrm{N}$-terminal and a $210-\mathrm{kDa} \mathrm{C}-$ terminal fragment as described previously. ${ }^{17}$ Both fragments were compared with intact fibronectin in terms of their capability to mediate staphylococcal 
adherence to fibrin clots and plastic surfaces. For this, adherence experiments were performed as described above with equimolar concentrations of fibronectin fragments and intact fibronectin, i.e., $0.68 \mu \mathrm{mol} / \mathrm{ml}$ for repletion of fibronectin-depleted plasma, $2 \cdot 26 \mu \mathrm{mol} / 10^{9}$ staphylococci for the pre-treatment of staphylococci before adherence, and $0.113 \mu \mathrm{mol} /$ well for the coating of plastic surfaces.

\section{Results}

\section{Binding of staphylococci to soluble fibronectin}

Binding was determined with soluble, ${ }^{125}$ I-labelled fibronectin purified from human plasma. Only $S$. aureus bound substantial amounts of fibronectin (18\% binding activity). None of the CNS tested had binding activities exceeding $5 \%$, which is generally considered as non-specific background binding.

\section{Adherence of staphylococci to fibrin thrombi}

The adherence of several potential wound pathogens to fibrin clots was compared. Substantial adherence was observed with $S$. aureus, S. epidermidis and Str. pyogenes $-60,58$ and $57\left(\times 10^{5}\right)$ adherent bacteria, respectively. $S$. intermedius and Str. agalactiae, species known to have very low binding activities for fibronectin, showed a much weaker adherence to fibrin thrombi (table).

\section{Role of fibronectin in staphylococcal adherence to fibrin thrombi}

Five randomly selected clinical isolates each of $S$. epidermidis and $S$. aureus were tested. All $S$. epidermidis isolates adhered significantly less to fibrin clots that had been prepared from fibronectin-depleted plasma than to clots prepared from normal plasma. Mean adherence reduction caused by fibronectin depletion was $45 \%$. Reduced adherence was almost completely restored after repletion with fibronectin (fig. 1a). However, the mean adherence of five $S$. aureus clinical isolates was almost identical in terms of fibronectin-depleted thrombi and clots prepared from normal or fibronectin-repleted plasma (fig. 1a). The effects of soluble fibronectin on staphylococcal adherence to fibrin thrombi were also studied, since fibronectin is available to bacteria as a component of wound exudates in vivo. Staphylococci were incubated with $100 \mu \mathrm{g}$ of plasma fibronectin $/ 10^{9}$ bacteria before adherence experiments. As expected, adherence of $S$. epidermidis, which did not bind fibronectin in the binding assays, was not affected by prior incubation with soluble plasma fibronectin. However, S. aureus showed an increase of adherence to fibrin thrombi after incubation with fibronectin (fig. 1 b).

In parallel experiments with incorporated as well as soluble fibronectin fragments, neither $29-\mathrm{kDa} \mathrm{N}$ terminal nor $210-\mathrm{kDa}$ C-terminal fragment had any significant effect on the adherence of either staphylococcal species to fibrin clots.
Role of fibronectin in staphylococcal adherence to plastic surfaces

The adherence of several CNS species was tested and a $90 \%$ reduction of "non-specific" adherence was observed when plates had been blocked with PBS containing BSA $1 \%$. After blocking, CNS adhered in numbers up to 10-fold higher to fibronectin-coated plates than to non-coated plates (fig. 2). Plates were coated with increasing fibronectin concentrations

Table. Adherence of bacteria to fibrin thrombi in vitro

\begin{tabular}{lcc}
\hline \multicolumn{1}{c}{ Species } & $\begin{array}{c}\text { Number of } \\
\text { isolates }\end{array}$ & $\begin{array}{c}\text { Mean numbers (SD) } \\
\text { of adherent bacteria } \\
\left(\times 10^{5}\right)\end{array}$ \\
\hline S. aureus & 10 & $60(14)$ \\
S. epidermidis & 10 & $58(16)$ \\
S. intermedius & 2 & $21(3 \cdot 5)$ \\
Str. pyogenes & 8 & $57(8)$ \\
Str. agalactiae & 2 & $18(1 \cdot 7)$ \\
\hline
\end{tabular}

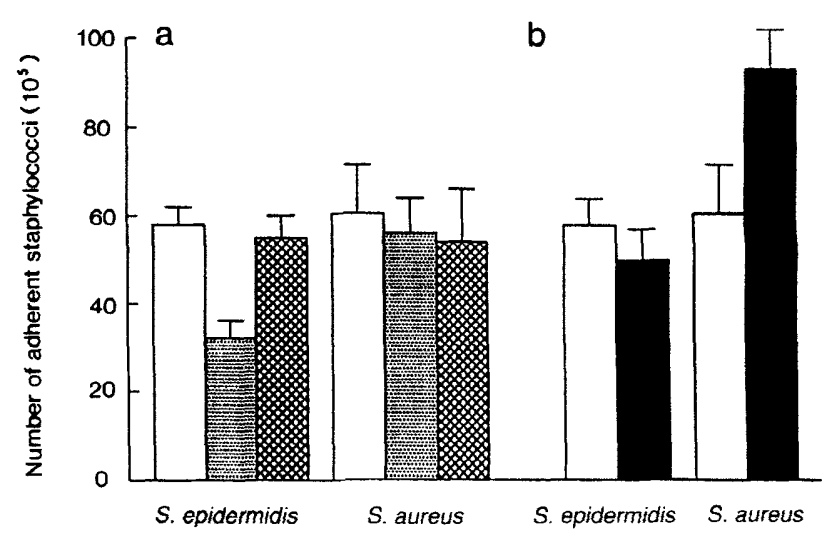

Fig. 1. Adherence of $S$. epidermidis and $S$. aureus to fibrin thrombi. (a) Thrombi were prepared from normal human plasma ( $\square$ ), plasma that had been depleted of fibronectin by affinity chromatography ( $\mathrm{Q})$ and repleted plasma ( $\otimes)$. (b) Adherence of $S$. epidermidis and $S$. aureus to fibrin thrombi after pre-incubation of bacteria with $100 \mu \mathrm{g}$ of purified soluble fibronectin for $45 \mathrm{~min}$ and washing with PBS ( $\square$ ) and without treatment with fibronectin (control, $\square$ ). Results were obtained from five randomly selected clinical isolates in each experiment and are expressed as mean numbers (SD) of adherent bacteria/clot.

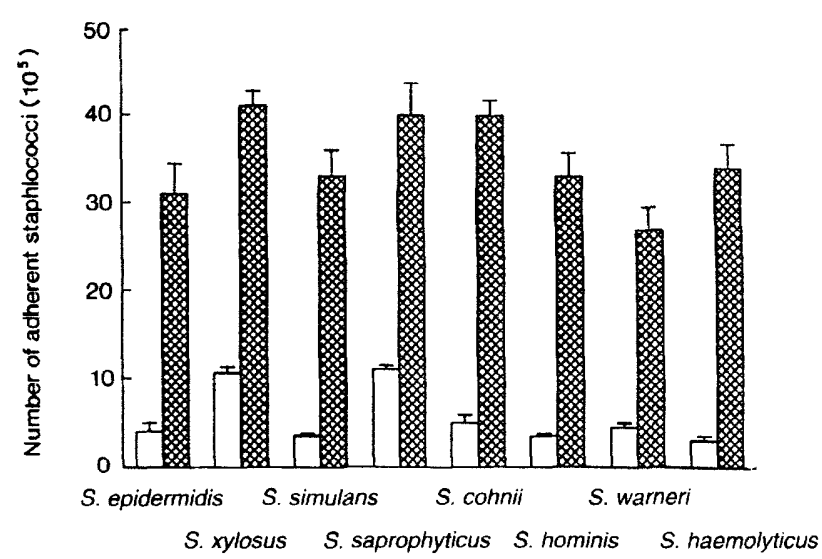

Fig. 2. Adherence of CNS to plastic surfaces coated with fibronectin $(B)$ and control surfaces $(\square)$. Each well of the microtitration plates was coated with $5 \mu \mathrm{g}$ of purified fibronectin and the adherence of eight different species of CNS was determined. Results were obtained from two strains of each species and are expressed as mean numbers (SD) of adherent bacteria. 


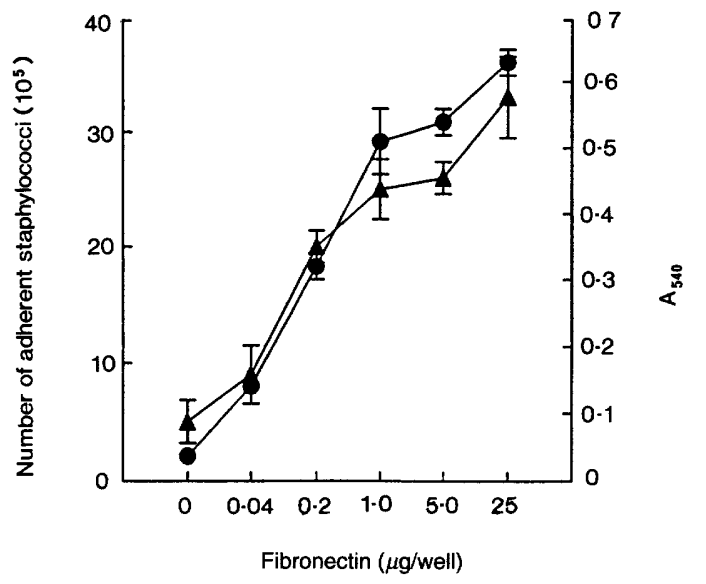

Fig. 3. Adherence of $S$. epidermidis strain CI 281 to plastic surfaces coated with fibronectin. Microtitration wells were coated with increasing concentrations (0.04-25 $\mu \mathrm{g} /$ well) of fibronectin and, after washing, amounts of fibronectin bound to the plastic were determined by ELISA. In parallel, the adherence of a representative $S$. epidermidis strain (CI 281) to coated wells was determined. Results are shown as, respectively, mean ELISA values at $540 \mathrm{~nm}\left(\mathrm{~A}_{540}\right.$; - -) and mean numbers of adherent staphylococci (SD) $(\boldsymbol{\Delta}-\boldsymbol{\Delta})$. Each assay was carried out in quadruplicate.

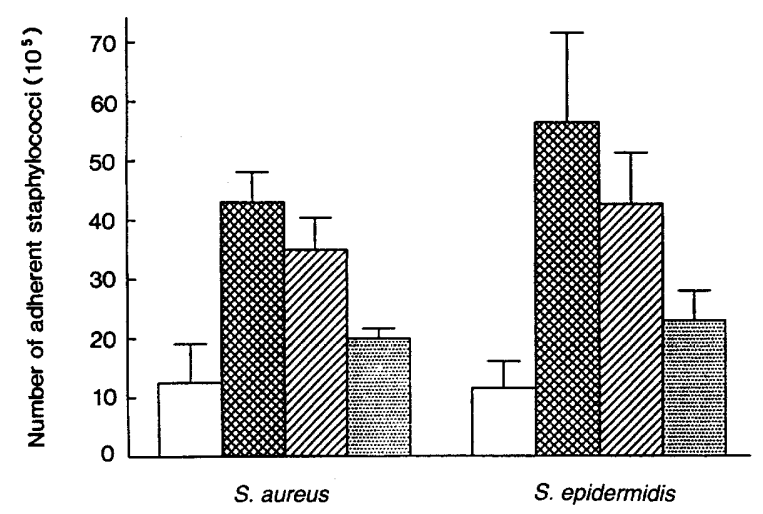

Fig. 4. Adherence of a representative strain each of $S$. aureus (strain Mi 32) and S. epidermidis (strain CI 281) to plastic surfaces coated with equimolar concentrations $(0.113 \mu \mathrm{mol} /$ well) of fibronectin ( $)$, 29-kDa N-terminal fragment ( $(2)$ or 210-kDa C-terminal fragment (圆) of fibronectin and to control (uncoated) surfaces $(\square)$. Adherence is expressed as mean number (SD) of adherent bacteria in four experiments.

$(0 \cdot 04-25 \mu \mathrm{g} /$ well $)$ to confirm that the increase of adherence was specifically mediated by fibronectin, and the adherence of $S$. epidermidis and amounts of fibronectin bound to plastic were determined in parallel. The increase of staphylococcal adherence and concentration of fibronectin immobilised on the plastic surface showed a strong correlation (fig. 3). Similar experiments were done with plates that had been coated with BSA at increasing concentrations and then treated with fibronectin, but no significant effect on the subsequent binding of fibronectin and adherence of $S$. epidermidis was observed. Results obtained by ELISA confirmed that BSA-coating of plastic surfaces reached saturation with $1 \mu \mathrm{g} /$ well, which is $0 \cdot 1 \%$ of the concentration used for blocking. It was also found that plasma coating of plastics was almost as effective as purified fibronectin in facilitating adherence of CNS (data not shown). With equimolar concentrations of fibronectin fragments used for coat- ing plastic surfaces, only the $29-\mathrm{kDa} \mathrm{N}$-terminal fragment enhanced adherence of CNS to plastic (fig. 4). Adherence experiments were also performed with PVC instead of polystyrene microtitration plates to compare the effects of different synthetic materials on the adherence of CNS. No difference was observed, except that CNS adherence was about two-fold higher to non-coated polystyrene than to PVC without BSA blocking.

Attachment of fibronectin, albumin, fibrinogen and $\alpha_{2}$ macroglobulin to plastic after plasma coating

Analysis by ELISA of plastic surfaces coated with human plasma $50 \mu \mathrm{l} /$ well showed that fibronectin, albumin, fibrinogen and $\alpha_{2}$ macroglobulin all bound to the plastic surface, with fibrinogen binding most and fibronectin binding least. However, in terms of the physiological concentrations of these plasma proteins, the fibronectin which bound to plastic represented $c$. $30 \%$ of total plasma fibronectin whereas the binding of fibrinogen was $10 \%$, and that of albumin and $\alpha_{2}$ macroglobulin was $5 \%$.

\section{Discussion}

In recent years, staphylococci have emerged as a cause of major nosocomial infections associated with post-operative complications and the use of intravenous catheters and prosthetic devices. The initiating event in such infections is the specific adherence of the organisms to blood clots and implanted or inserted foreign materials. A number of adhesive plasma proteins such as fibronectin, fibrinogen and vitronectin which are present on many host cell surfaces have been proposed as bridging molecules in the adherence of pathogenic cocci. ${ }^{20-22}$ These proteins are also major components of fibrin clots formed at an injured site and may form a "conditioning layer" on the surface of synthetic materials exposed to blood or tissue fluids after implantation or insertion. ${ }^{6,23.24}$ Recently, a mediatory role of fibronectin in streptococcal adherence to fibrin thrombi was demonstrated ${ }^{16}$ but this study could not confirm the results of Toy et al. ${ }^{14}$ on the involvement of fibronectin in S. aureus adherence. In both studies fibronectin incorporated into the clot was used, but not soluble fibronectin. However, it is known that fibronectin immobilised on surfaces may exhibit binding characteristics for bacteria that differ from those of soluble fibronectin. ${ }^{8,13}$ In the present study, it was shown that CNS, although considered to be unable to bind to fibronectin, possessed significant affinity for fibronectin incorporated into fibrin thrombi or immobilised on plastic surfaces. Soluble fibronectin had no effect on CNS adherence to fibrin clots or plastic. In contrast, all clinical isolates of $S$. aureus tested bound soluble fibronectin, and pretreatment with soluble fibronectin increased their adherence to fibrin thrombi. Interestingly, S. aureus 
showed almost no binding to fibronectin incorporated into clots. The different binding characteristics of CNS and $S$. aureus for soluble versus incorporated and immobilised fibronectin presumably reflects conformational differences, since soluble fibronectin appears to be a globular protein, whereas during immobilisation the two arms of the fibronectin dimer unfold completely. ${ }^{25}$

Like most fibronectin-binding bacteria, $S$. aureus recognises the $\mathrm{N}$-terminal region of fibronectin, although it also binds to a second domain in the $\mathrm{C}$ terminal part. ${ }^{26}$ Fibronectin can be cleaved by thrombin into two fragments, a $29-\mathrm{kDa} \mathrm{N}$-terminal and a $210-\mathrm{kDa} \mathrm{C}$-terminal fragment, each of which contains one of the binding sites. ${ }^{17}$ Such fragments were used to localise the binding site responsible for the adherence of staphylococci to fibronectin in fibrin clots and immobilised on plastic. It was found that when immobilised on to plastic surfaces, only the 29 $\mathrm{kDa} \mathrm{N}$-terminal fragment enhanced adherence of both CNS and $S$. aureus to almost the same extent as intact fibronectin. This indicates that both staphylococcal species might interact with similar binding sites in fibronectin immobilised on to plastic surfaces. However, neither incorporated nor soluble 29-kDa fragment affected staphylococcal adherence to fibrin clots, which suggests that the $29-\mathrm{kDa}$ fragment might exhibit different conformational and binding characteristics, as has been discussed in a recent study on intact fibronectin. ${ }^{13}$ In vivo, implanted or inserted synthetic materials are readily covered by plasma proteins. ${ }^{24}$ Therefore, it was of interest to determine whether plasma fibronectin would be available in sufficient amounts after plasma coating of plastics. We found by ELISA that c. $30 \%$ of the fibronectin normally present in plasma remains bound to plastic surfaces after plasma coating. Other major plasma proteins such as albumin, fibrinogen or $\alpha_{2}$ macroglobulin bound to a much lower extent in relation to their physiological concentrations. Since albumin is a major blood protein which might influence coating of implanted synthetic

\section{References}

1. Pfaller MA. Herwaldt LA. Laboratory, clinical, and epidemiological aspects of coagulase-negative staphylococci. Clin Microbiol Rev 1988; 1 : 289-299.

2. Wadström T. Molecular aspects on pathogenesis of wound and foreign body infections due to staphylococci. Zentralbl Bakteriol Mikrobiol Hy A 1987; 266: 191-211.

3. Maki DG. Infections associated with intravascular lines. In: Remington JS, Swartz MN (eds) Current clinical topics in infectious diseases. New York, McGraw-Hill. 1982: 309.

4. Beachey EH. Bacterial adherence : adhesin-receptor interactions mediating the attachment of bacteria to mucosal surfaces J Infect Dis 1981: 143: 325-345.

5. Grinnell F. Feld MK. Distribution of fibronectin on peripheral blood cells in freshly clotted blood. Thromb Res 1981: 24 : $397-404$.

6. Vaudaux P, Suzuki R, Waldvogel FA, Morgenthaler JJ, Nydegger UE. Foreign body infection: role of fibronectin as a ligand for the adherence of Staphylococcus aureus. $J$ Infect Dis 1984: 150: 546-553.

7. Kuusela P. Vartio T. Vuento M, Myhre EB. Binding sites for streptococci and staphylococci in fibronectin. Infect Immun $1984 ; 45: 433-436$. materials by fibronectin and adherence of staphylococci in vivo, the effects of albumin on fibronectin coating and adherence were further analysed. In agreement with other studies, ${ }^{9,27}$ we observed that albumin had a very significant blocking effect on staphylococcal attachment to non-coated plastics. However, fibronectin bound to nearly the same extent to albumin-coated and non-coated plastic, thereby permitting a similar increase of CNS adherence. Recently, Vaudaux and co-workers reported that different inserted materials reacted similarly with respect to their coating by host proteins. ${ }^{6} \mathrm{We}$ also found no differences between polystyrene tissue culture plates and PVC plates (which were compared in some experiments), except that without prior BSA blocking, staphylococcal adherence to non-coated surfaces was up to two times higher with polystyrene than with PVC plates. Recently, Muller et al. ${ }^{28}$ reported that they could not observe a role of plasma proteins in adherence of CNS to plastics. However, their adherence studies were done without the albumin normally present under physiological conditions. In the absence of albumin, CNS show such a strong adherence to plastics that quantification of adherencemediating effects of plasma proteins may be very difficult. Our results showed that CNS, although generally known to have almost no affinity for soluble fibronectin, can adhere to blood clots and synthetic materials through interactions with immobilised fibronectin. This could be of importance for the initiation of catheter-related CNS infections since implanted or inserted materials may cause blood clotting and are readily covered by plasma proteins such as fibronectin. On the other hand, the preferential adherence of $S$. aureus for blood clots through soluble fibronectin suggests that these bacteria as primary wound pathogens might use soluble fibronectin present in wound exudates for their adherence to an injured site.

This work was supported by grant $01 \mathrm{KI} 88147$ from the Bundesministerium für Forschung und Technologie, Bonn, Germany.

8. Kuusela P, Vartio T, Vuento M, Myhre EB. Attachment of staphylococci and streptococci on fibronectin, fibronectin fragments and fibrinogen bound to a solid phase. Infect Immun 1985; 50: 77-81.

9. Vaudaux P, Pittet D, Haeberli A et al. Host factors selectively increase staphylococcal adherence on inserted catheters: a role for fibronectin and fibrinogen or fibrin. I Infect Dis 1989; 160: 865-875

10. Simpson WA, Beachey EH. Adherence of group A streptococci to fibronectin on oral epithelial cells. Infect Immun 1983; 39: 275-279.

11. Myhre EB, Kuusela P. Binding of human fibronectin to group A, C, and G streptococci. Infect Immun 1983; 40: 29-34.

12. Doran JE, Raynor RH. Fibronectin binding to protein Acontaining staphylococci. Infect Immun $1981 ; 33$ : 683-689.

13. Raja RH, Raucci G, Hook M. Peptide analogs to a fibronectin receptor inhibit attachment of Staphylococcus aureus to fibronectin-containing substrates. Infect Immun 1990; 58: 2593-2598.

14. Toy PTCY, Lai L-W, Drake TA, Sande MA. Effect of fibronectin in adherence of Staphylococcus aureus to fibrin thrombi in vitro. Infect Immun 1985; 48: 83-86.

15. Mertz PM, Patti JM, Marcin JJ, Marshall DA. Model for 
studying bacterial adherence to skin wounds. $J$ Clin Microbiol 1987; 25: 1601-1604.

16. Chhatwal GS, Valentin-Weigand P, Timmis KN. Bacterial infection of wounds: fibronectin-mediated adherence of group $\mathrm{A}$ and $\mathrm{C}$ streptococci to fibrin thrombi in vitro. Infect Immun 1990; 58: 3015-3019.

17. Chhatwal GS, Blobel H. Heterogeneity of fibronectin reactivity among streptococci as revealed by binding of fibronectin fragments. Comp Immunol Microbiol Infect Dis 1987; 10: 99-108.

18. Miekka SI, Ingham KC, Menache D. Rapid methods for isolation of human plasma fibronectin. Thromb Res 1982; 27: 1-4.

19. Hunter WM, Greenwood FC. Preparation of iodine-131 labelled human growth hormone of high specific activity. Nature 1962; 194: 495-496.

20. Valentin-Weigand P, Grulich-Henn J, Chhatwal GS, MüllerBerghaus G, Blobel H, Preissner KT. Mediation of adherence of streptococci to human endothelial cells by complement $\mathrm{S}$ protein (Vitronectin). Infect Immun 1988; 56: 2851-2855.

21. Cheung AL, Fischetti VA. The role of fibrinogen in staphylococcal adherence to catheters in vitro. J Infect Dis $1990 ; 161$ : 1177-1186.
22. Vercellotti GM, Lussenhop D, Peterson PK et al. Bacterial adherence to fibronectin and endothelial cells: a possible mechanism for bacterial tissue tropism. J Lab Clin Med $1984 ; 103: 34-43$.

23. Grinnel F, Billingham RE, Burgess L. Distribution of fibronectin during wound healing in vivo. J Invest Dermatol 1981; 76: 181-189.

24. Baier RE. The organization of blood components near interfaces. Ann NY Acad Sci 1977; 283: 17-36.

25. Proctor RA. Fibronectin: a brief overview of its structure, function, and physiology. Rev Infect Dis 1987; 9 Suppl 4: S317-S321.

26. Höök M, Switalski LM, Wadström T, Lindberg M. Interactions of pathogenic microorganisms with fibronectin. In: Mosher DF (ed) Fibronectin. London, Academic Press Inc. 1989: 295-308.

27. Pascual A, Fleer A, Westerdaal NAC, Verhoef J. Modulation of adherence of coagulase-negative staphylococci to teflon catheters in vitro. Eur J Clin Microbiol 1986; 5: 518-522.

28. Muller E, Takeda S, Goldmann DA, Pier GB. Blood proteins do not promote adherence of coagulase-negative staphylococci to biomaterials. Infect Immun 1991; 59: 3323-3326. 